\title{
Bronchoscopically delivered microwave ablation in an in vivo porcine lung model
}

\author{
Jan Sebek ${ }^{1,2}$, Steve Kramer ${ }^{3}$, Rob Rocha ${ }^{3}$, Kun-Chang Yu ${ }^{3}$, Radoslav Bortel ${ }^{2}$, \\ Warren L. Beard ${ }^{4}$, David S. Biller ${ }^{4}$, David S. Hodgson ${ }^{4}$, Charan K. Ganta ${ }^{5}$, \\ Henky Wibowo ${ }^{6}$, John Yee ${ }^{7}$, Renelle Myers ${ }^{7,8}$, Stephen Lam ${ }^{8}$ and Punit Prakash ${ }^{1}$
}

Affiliations: ${ }^{1}$ Dept of Electrical and Computer Engineering, Kansas State University Manhattan, Manhattan, KS, USA. ${ }^{2}$ Dept of Circuit Theory, Czech Technical University in Praque, Prague, Czech Republic. ${ }^{3}$ Broncus Medical, Inc., San Jose, CA, USA. ${ }^{4}$ Dept of Clinical Sciences, Kansas State University, Manhattan, KS, USA. ${ }^{5}$ Dept of Diagnostic Medicine/Pathobiology, Kansas State University, Manhattan, KS, USA. ${ }^{6}$ Phenomapper, LLC., San Jose, CA, USA. ${ }^{7}$ Dept of Thoracic Surgery, Vancouver General Hospital and the University of British Columbia, Vancouver, Canada. ${ }^{8}$ Dept of Integrative Oncology, BC Cancer Research Center and the University of British Columbia, Vancouver, Canada.

Correspondence: Punit Prakash, Department of Electrical and Computer Engineering, Kansas State University, 3078 Engineering Hall, 1701D Platt St, Manhattan, KS 66506, USA. E-mail: prakashplaksu.edu

\section{ABSTRACT}

Background: Percutaneous microwave ablation is clinically used for inoperable lung tumour treatment. Delivery of microwave ablation applicators to tumour sites within lung parenchyma under virtual bronchoscopy guidance may enable ablation with reduced risk of pneumothorax, providing a minimally invasive treatment of early-stage tumours, which are increasingly detected with computed tomography (CT) screening. The objective of this study was to integrate a custom microwave ablation platform, incorporating a flexible applicator, with a clinically established virtual bronchoscopy guidance system, and to assess technical feasibility for safely creating localised thermal ablations in porcine lungs in vivo.

Methods: Pre-ablation CTs of normal pigs were acquired to create a virtual model of the lungs, including airways and significant blood vessels. Virtual bronchoscopy-guided microwave ablation procedures were performed with 24-32 W power (at the applicator distal tip) delivered for 5-10 mins. A total of eight ablations were performed in three pigs. Post-treatment CT images were acquired to assess the extent of damage and ablation zones were further evaluated with viability stains and histopathologic analysis.

Results: The flexible microwave applicators were delivered to ablation sites within lung parenchyma 5-24 mm from the airway wall via a tunnel created under virtual bronchoscopy guidance. No pneumothorax or significant airway bleeding was observed. The ablation short axis observed on gross pathology ranged $16.5-23.5 \mathrm{~mm}$ and $14-26 \mathrm{~mm}$ on CT imaging.

Conclusion: We have demonstrated the technical feasibility for safely delivering microwave ablation in the lung parenchyma under virtual bronchoscopic guidance in an in vivo porcine lung model.

@ERSpublications

This paper demonstrates the technical feasibility of safely delivering microwave ablation in the lung parenchyma under virtual bronchoscopic guidance in an in vivo porcine lung model https://bit.ly/32aruLf

Cite this article as: Sebek J, Kramer S, Rocha R, et al. Bronchoscopically delivered microwave ablation in an in vivo porcine lung model. ERJ Open Res 2020; 6: 00146-2020 [https://doi.org/ 10.1183/23120541.00146-2020]. 


\section{Introduction}

While curative surgical resection [1] remains the gold standard treatment for patients with early-stage lung cancer, only about $30 \%$ of patients are surgical candidates due to comorbid disease, cardiopulmonary function, or tumour location within the lung $[2,3]$. Alternatives to surgery are chemotherapy, stereotactic body radiation therapy (SBRT), or percutaneous thermal ablation modalities. SBRT is the most widely used; while recent studies have demonstrated good local control with SBRT, some patients are ineligible due to prior ionising radiation exposure or tumour location within the lung [4]. Several thermal ablation modalities (radiofrequency (RF) ablation, microwave ablation (MWA), cryoablation) have been applied clinically with needle-based applicators inserted into the lung via an image-guided transcutaneous approach. A recent multi-centre prospective study reported similar 2-year overall survival rate for patients with stage IA nonsmall cell lung cancer treated with SBRT and percutaneous MWA [5]. Unlike SBRT, thermal ablation does not employ ionising radiation and thus has a limited toxicity burden, and is amenable to repeat procedures [6,7]. While RF ablation is currently the most common thermal ablation modality [7], MWA offers several advantages for treating lung tumours: microwaves can propagate through aerated lung and charred tissue that may form around the applicator, enabling use of simplified energy-delivery algorithms; and provide higher heating rates over a broader tissue area, and can thus more readily overcome heat sinks due to blood perfusion, airflow in large airways, and ventilation [8].

Although percutaneous thermal ablation approaches have shown promise in clinical use, a significant complication is the risk of pneumothorax [9]. An experimental study in a porcine model demonstrated that the creation of an iatrogenic fistula extending from the ablation zone to the pleural space is the likely cause of pneumothorax after percutaneous ablation [10]. Bronchoscopic transparenchymal nodule approaches [11] provide safe access to centrally located lung targets and may reduce the risk of pneumothoraces, when compared to percutaneous approaches, as has been demonstrated during biopsy procedures $[12,13]$.

We have recently reported on the development of a flexible, water-cooled MWA catheter for thermal ablation of lung tumours [14]. The objective of the present study was to integrate the MWA catheter and energy-delivery system with a clinically established bronchoscopic treatment planning, guidance, and navigation platform, and to assess the technical feasibility of performing ablation with this system in an in vivo nonsurvival porcine lung model. The extents of the ablation zone were assessed with post-ablation computed tomography (CT) imaging and viability staining, and characteristics of the ablation zone were assessed following histopathologic analysis.

\section{Methods}

Bronchoscopic microwave ablation system

We used a custom $1.1 \mathrm{~m}$ long, $1.8 \mathrm{~mm}$ outer diameter, water-cooled MWA catheter (figure 1), adapted from the design previously described by Pfannenstiel et al. [14]. Microwave power at $2.45 \mathrm{GHz}$ was coupled to the catheter distal tip via a flexible coaxial cable. Due to cable attenuation, approximately $52 \%$ of the power applied at the catheter input was delivered to the distal tip. Chilled water was circulated through the catheter to remove waste heat due to attenuation within the coaxial cable. An antenna that radiates microwaves into surrounding tissue was formed at the distal tip with a balun to restrict radiation along the applicator axis.
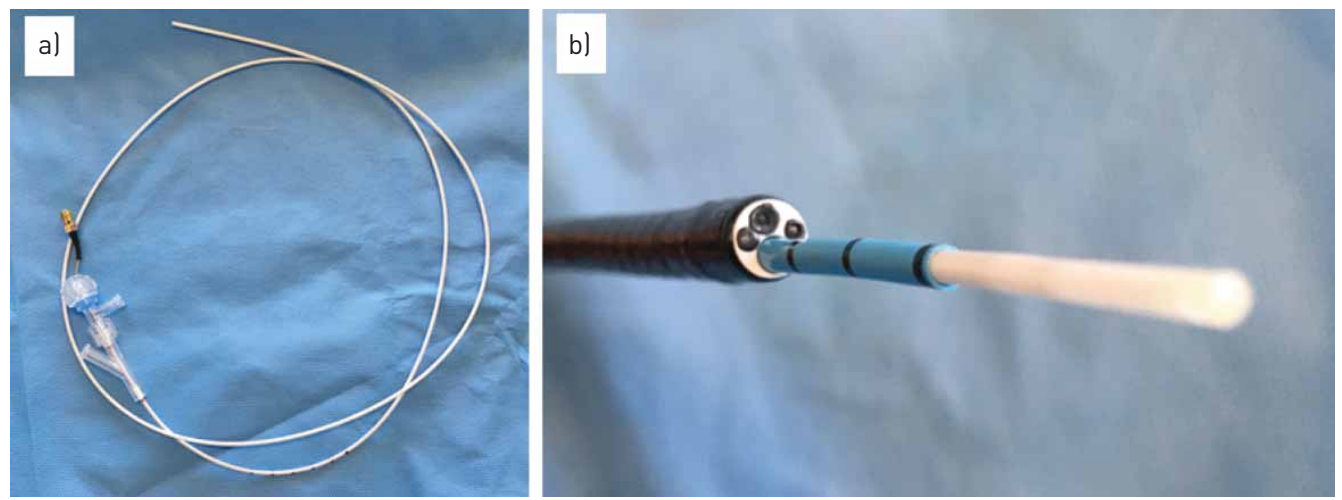

FIGURE 1 a) Flexible water-cooled microwave catheter. b) Distal tip of the catheter emerging from the instrument channel of a flexible bronchoscope, from within a guidance sheath. 
The MWA catheter was delivered to diverse sites within porcine lung through a flexible bronchoscope (Olympus BF 1T160) with guidance provided by the Archimedes treatment planning and navigation system. Microwave power was supplied by a solid-state $2.45 \mathrm{GHz}$ generator (GMS $200 \mathrm{~W}$, SAIREM). The generator was connected to the catheter via $10 \mathrm{ft}$ of RG-393 cable, with an inline power meter (Bird 7022). A peristaltic pump (Masterflex L/S7015-20) was used to circulate chilled water at $4{ }^{\circ} \mathrm{C}$ through the catheter. The integrated system for bronchoscopic ablation is illustrated in figure 2, with virtual bronchoscopy navigation using the Archimedes total lung access platform (Broncus Medical, Inc., San Jose, CA, USA.) $[12,15]$

\section{Animals and anaesthesia}

All experiments were conducted under a protocol approved by Kansas State University's Institutional Animal Care and Use Committee. Bronchoscopy-guided MWA was performed in domestic female pigs ( $\mathrm{n}=3$; weight: $45-50 \mathrm{~kg}$ ). Planning CT images were acquired 1-4 days prior to the MWA procedures to generate a virtual airway and blood vessel map using the Archimedes virtual bronchoscopy system. For both the imaging and ablation procedures, anaesthesia was induced with Telazol $\left(4.4 \mathrm{mg} \cdot \mathrm{kg}^{-1}\right.$, intramuscular) and xylazine $\left(2.2 \mathrm{mg} \cdot \mathrm{kg}^{-1}\right.$, intramuscular $)$ and followed with atropine sulfate $\left(0.05 \mathrm{mg} \cdot \mathrm{kg}^{-1}\right.$, intramuscular) to aid intubation. Anaesthesia was maintained by isoflurane at $1.5-3.0 \%$ and oxygen. During ablation procedures, two pigs were allowed to breathe spontaneously; one pig was ventilated by intermittent positive-pressure ventilation (I:E ratio 1:2, respiratory rate $16-20 / \mathrm{min}$, tidal volume $60-90 \mathrm{~mL}$ ) to maintain end-tidal $\mathrm{CO}_{2}\left(P_{\mathrm{ETCO}_{2}}\right)<55 \mathrm{mmHg}$. Pigs were instrumented for continuous monitoring of ECG, $P_{\mathrm{ETCO}_{2}}$, arterial oxygen saturation measured by pulse oximetry $\left(S_{\mathrm{pO}_{2}}\right)$ and respiratory rate. After completion of ablation procedures and post-procedure imaging, animals were euthanised under anaesthesia with $10 \mathrm{~mL}$ pentobarbital $\left(390 \mathrm{mg} \cdot \mathrm{mL}^{-1}\right)$ administered intravenously; euthanasia was administered within 60 min of the last ablation performed in each animal.

\section{Virtual bronchoscopy-guided microwave ablation procedures}

Pre-procedural CT images (GE Brightspeed, 16 slices, $120 \mathrm{kV}, 240-300 \mathrm{~mA}, 0.625 \mathrm{~mm}$ slice thickness) of inflated lungs were acquired with anesthetised pigs positioned in a dorsal recumbency to facilitate treatment planning for virtual bronchoscopy using the Archimedes system. Pigs were rapidly, manually

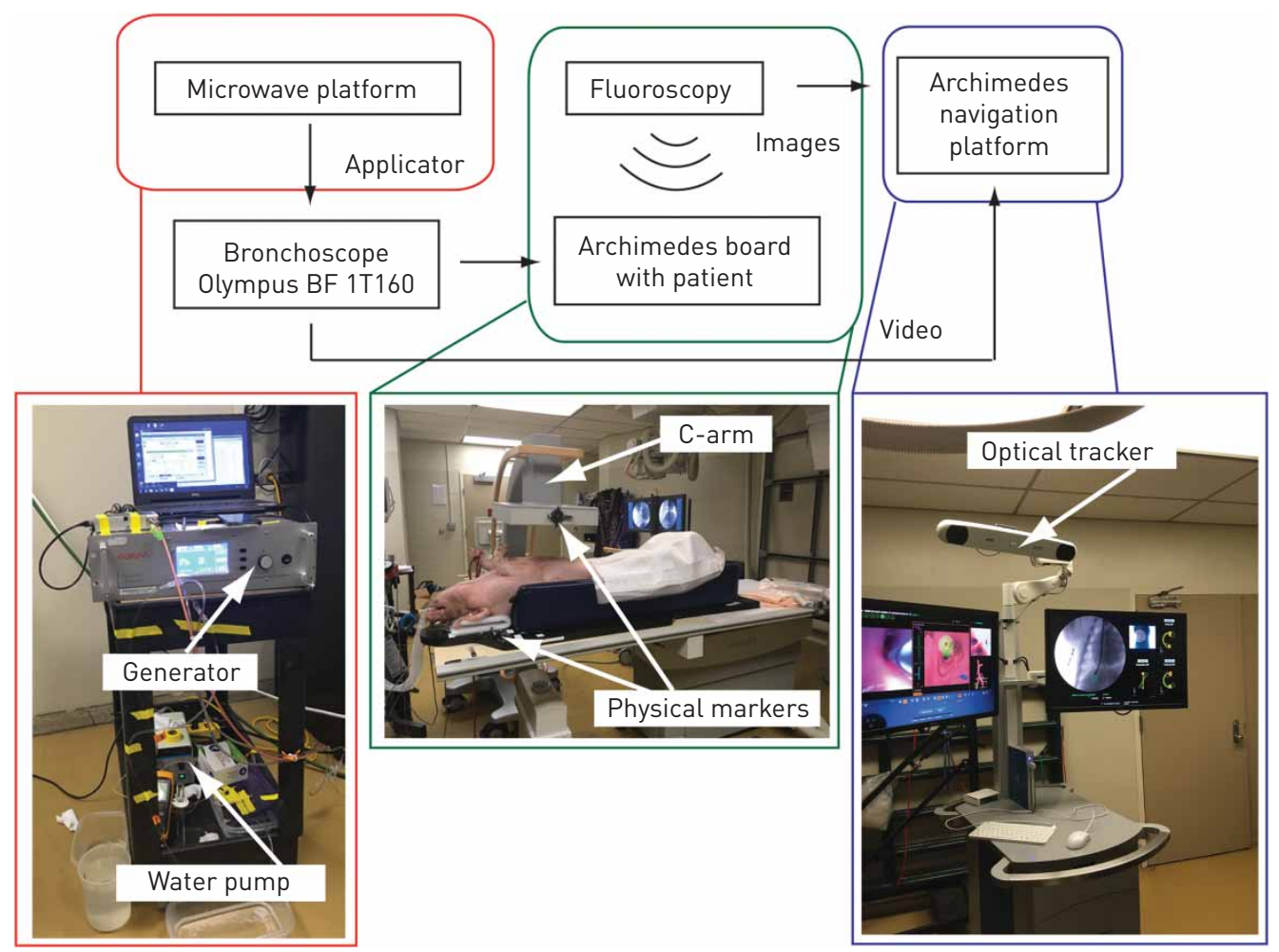

FIGURE 2 System for bronchoscopic delivery of microwave ablation. Components include: flexible microwave catheter; microwave generator and pump for catheter cooling; Archimedes treatment planning, guidance and navigation system. 
ventilated for approximately 15 breaths to induce apnoea and maintained at a positive end-expiratory pressure of $12 \mathrm{~cm} \mathrm{H}_{2} \mathrm{O}$ during image acquisition. Airways and blood vessels were automatically segmented within Archimedes, and used to create a virtual map of the vasculature and airways within the porcine lungs. Virtual ablation targets were positioned at diverse lung sites as shown in figure 3. The Archimedes system proposes a path through the airways to guide the bronchoscope to a point of entry (POE) into the parenchyma. Starting from this POE, a path is proposed by which the bronchoscopist can create a tunnel to access the target using the Archimedes Airway Access Kit, while minimising the risk of traversing blood vessels $[12,15]$.

Bronchoscopy-guided MWA procedures were carried out by a board-certified veterinary surgeon (W.L.B.) 1-4 days following pre-treatment imaging. Pigs were orotracheally intubated and positioned in dorsal recumbency. An Olympus BF-1T160 bronchoscope was passed into the trachea via the lumen of the endotracheal tube. A $90-\mathrm{cm}$ flexible sheath with stylet was inserted into the biopsy channel of the bronchoscope and positioned at the tip of the bronchoscope. As the present study was conducted in normal porcine lung without tumours, ablation sites were chosen in plausible tumour locations within diverse lung lobes, including sites in proximity to heatsinks such as large airways and vessels. For each target, the bronchoscopist used the Archimedes navigation to guide the bronchoscope to the POE identified during treatment planning. An 18-gauge flexible guarded needle (FlexNeedle) was advanced through the sheath's lumen until visible in the endoscopic field. The needle was deployed and used to puncture the airway into the lung parenchyma, and the sheath was then advanced over the needle until it entered the parenchyma. The FlexNeedle was withdrawn from the sheath and replaced by the stylet. The flexible sheath was advanced into the parenchyma to the ablation target site under Archimedes virtual bronchoscopy and fluoroscopy guidance. The stylet was then removed and replaced by the ablation catheter. Proper positioning of the ablation catheter was confirmed by real-time fused fluoroscopy [16] (see figure 4).

After confirming microwave applicator positioning, chilled water was circulated through the catheter and microwave power was applied. Two ablative doses were considered: $30-32 \mathrm{~W}$ for $5 \mathrm{~min}(\mathrm{n}=4)$, and $24 \mathrm{~W}$ for $10 \mathrm{~min}(\mathrm{n}=4)$. These power levels refer to power delivered to the distal tip of the catheter, and correspond to $40 \mathrm{~W}$ and $60 \mathrm{~W}$, respectively, at the catheter's proximal end. A maximum of $\mathrm{n}=4$ ablations were performed in each pig.

\section{Characterisation of ablation zone extents}

Post-procedure CT scans were taken before and following administration of $60 \mathrm{~mL}$ Omnipaque 300 injected via an ear vein. Ablation zones were segmented and assessed by a board-certified veterinary radiologist (D.S.B.). After euthanasia, the lungs were harvested and cut into 5-mm thick sections, which were immersed into triphenyl tetrazolium chloride, a viability stain that identifies cells with functioning mitochondria [17]. After staining, photographs of ablation zones were taken, and minimum and maximum diameters of each ablation zone were measured for the central region ("brown zone") as well as outer hyperaemic rim, consistent with published guidelines, as illustrated in figure 5 [18]. Extents of the ablation zones achieved in both experimental groups were statistically compared using ANOVA with Scheffe's post hoc test at a 5\% significance level.

\section{Histopathologic assessment of ablation zones}

Lung sections were fixed in $10 \%$ neutral buffered formalin, allowed to fix for $24 \mathrm{~h}$, and routinely processed for histopathological examination in an automated tissue processor and embedded in paraffin. Slide-mounted tissue sections $(\sim 4-\mu \mathrm{m}$ thick) were stained with haematoxylin and eosin $(\mathrm{H} \& \mathrm{E})$ and evaluated by a board-certified veterinary anatomic pathologist (C.K.G.).

\section{Results}

A total of eight virtual bronchoscopy-guided ablations were performed in three pigs. Estimates of the ablation zone extent recorded on gross pathology following vital staining and CT imaging of regions within the porcine lung, where ablations were performed, are reported in table 1 . ANOVA tests at a $5 \%$ significance level indicated that there was no statistically significant difference in ablation zone extents between the 5- and 10-min ablation groups. One ablation was performed without penetrating through the airway wall leaving the applicator inside a small airway; we estimate the applicator was in contact with the airway wall without any appreciable gap. Across all animals, no pneumothoraces were observed.

Figure 6 illustrates examples of ablation zones observed on CT imaging, gross pathology (following vital staining) and histopathology (H\&E-stained sections), following ablations at the two applied energy levels considered in this study. On histopathology, five distinct regions were observed, as further illustrated in figure 7 . 

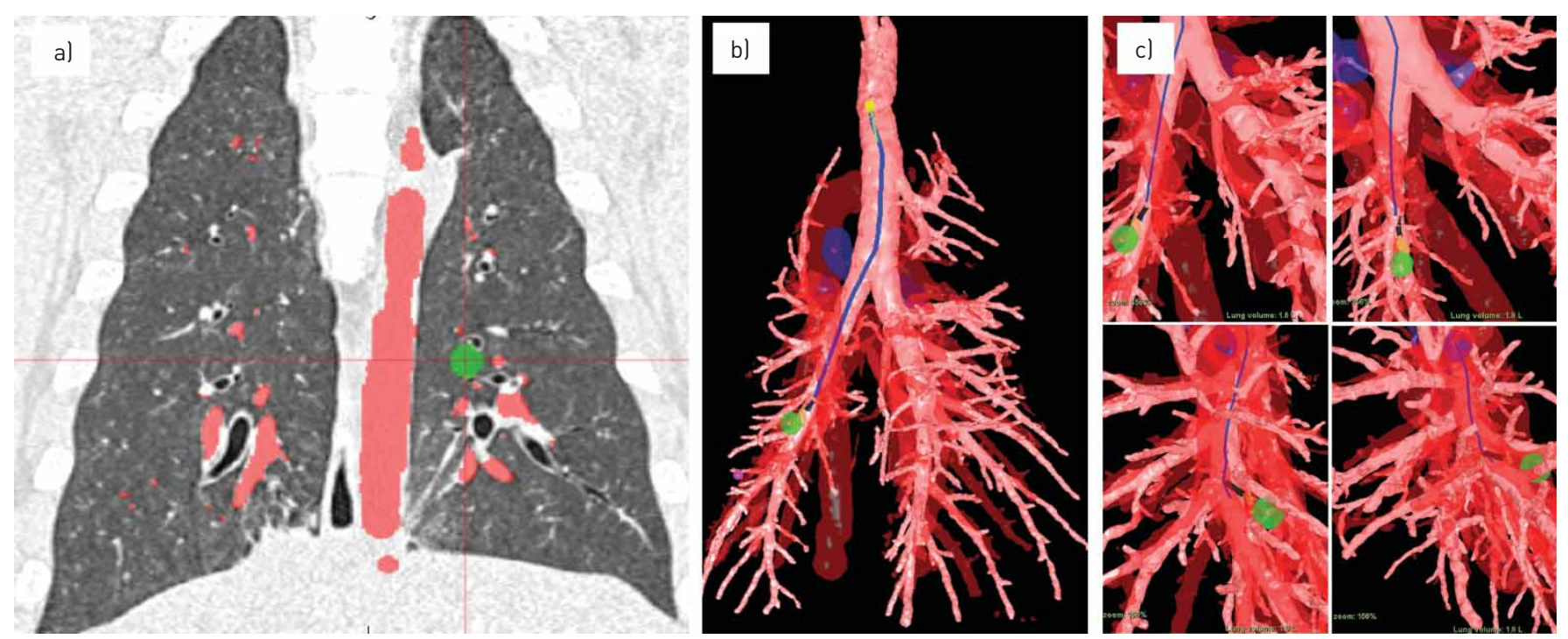

FIGURE 3 Example of planned target (green sphere) as seen on (a) pre-operative computed tomography image, (b) virtual map of lung airways and vessels with pathway how to access it (blue line), and (c) several close-up views of virtual map of airway and vessels, illustrating a path for accessing the target (green) from the airways.

Gross examination of the lung showed multiple targetoid lesions in the lung parenchyma following ablation (figure 6). The H\&E-stained sections of these areas showed a central area of necrosis (zone 1) that was characterised by marked disruption of tissue architecture, hypereosinophilia and nuclear pyknosis (necrosis) admixed with oedema and haemorrhage. Zone 2 showed marked alveolar oedema characterised

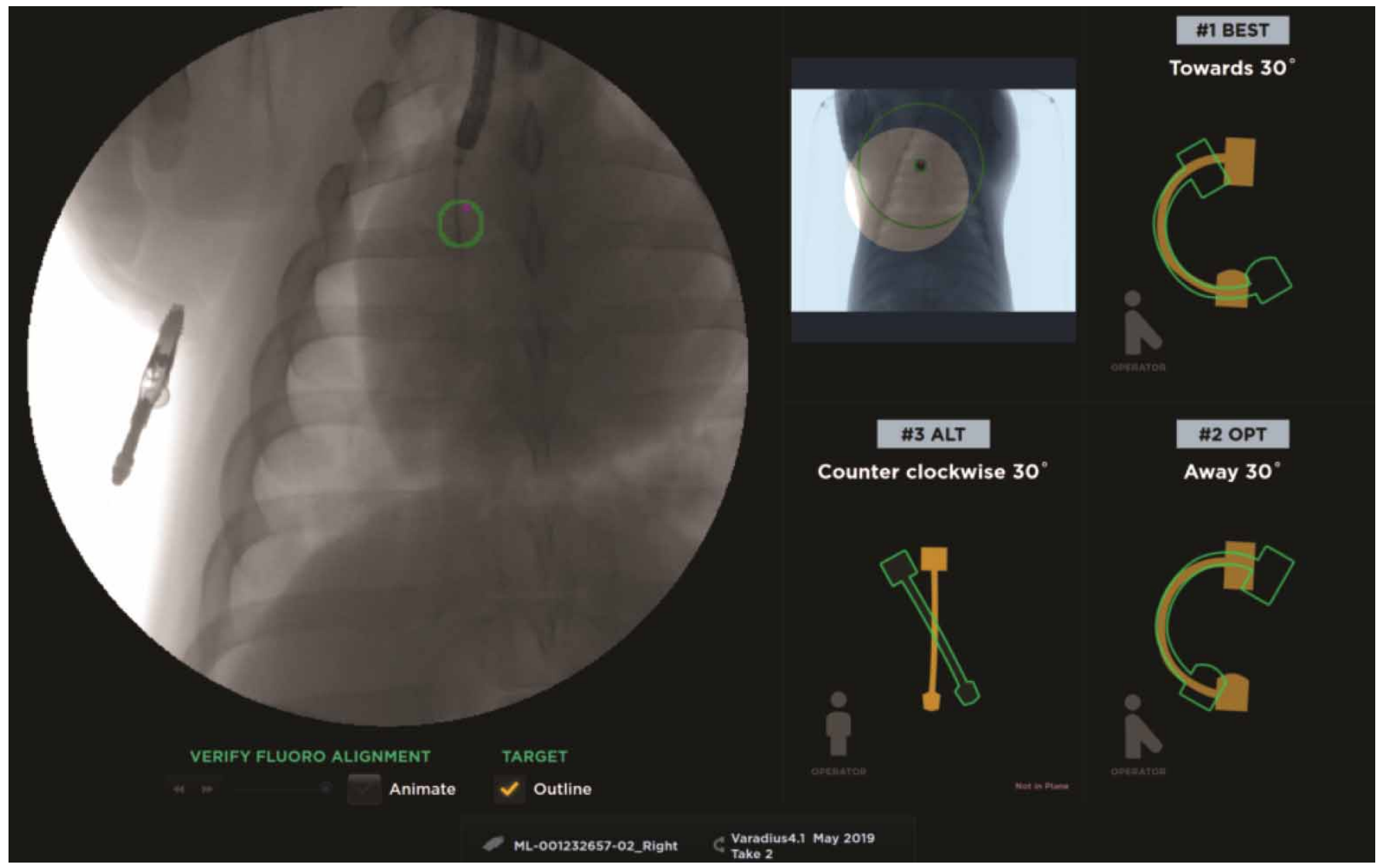

FIGURE 4 Example of fused fluoroscopy-based verification of microwave applicator placement within the targeted lung region. 
FIGURE 5 Example of ablation zone assessment in terms of maximum and minimum dimensions of central brown region as well as outer hyperaemic rim.

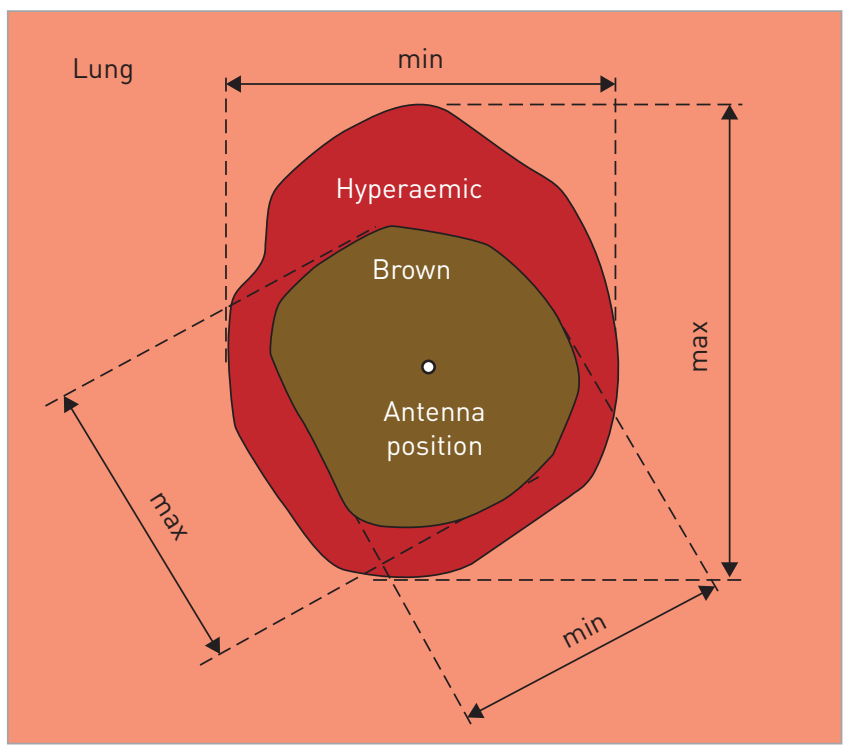

by pale eosinophilic proteinaceous material (oedema), zone 3 had mild oedema with often marked dilation of the airways (emphysema), zone 4 had marked vascular congestion/hyperaemia with mild interstitial haemorrhage and zone 5 had marked infiltration of macrophages and neutrophils, forming a concentric inflammatory cell cuff in this zone. Zone 6 represents unaffected lung parenchyma with no microscopic lesions. There is no microscopic evidence of vascular rupture of arteries and veins in both ablated and nonablated zones. The small blood vessels in the ablated zones 1-3 showed separation of vascular endothelium in both the veins and arteries. The vascular endothelial separation is noticed more frequently in veins and occasionally in arteries in zone 4 . No differences in the histological characteristics of tissue within zones 1-5 were observed for the ablations conducted after penetrating the airway wall into the lung parenchyma compared with the applicator within the airway.

\section{Discussion}

Pneumothorax is the most common and a substantial risk for patients treated with percutaneous MWA, and treatment of centrally located tumours may be technically challenging [10]. The present study was designed to assess the technical feasibility of ablating lung tissue in an in vivo porcine model delivered with a bronchoscopic MWA system integrated with a clinically established virtual bronchoscopy guidance, treatment planning and navigation platform. No cases of pneumothorax were observed in this study, demonstrating the feasibility of safely delivering bronchoscopic MWA.

Previously published pre-clinical studies of microwave systems for lung ablation have been restricted to needle-based applicators delivered using an open surgical or percutaneous approach $[17,19,20]$. In the

TABLE 1 Estimated dimensions of ablation zones in a plane perpendicular to the applicator insertion along with mean values and SD

\begin{tabular}{|c|c|c|c|c|c|c|c|c|c|}
\hline Power W & Time $\min$ & \multicolumn{2}{|c|}{ Brown region } & \multicolumn{2}{|c|}{ Hyperaemic region } & \multicolumn{3}{|c|}{ CT dimensions } & Lung lobe and tunnel length $\mathrm{mm}$ \\
\hline 32 & 5 & 11 & 13 & 16 & 22 & 15.4 & 24.5 & 21 & Right cranial, 11.5 \\
\hline 32 & 5 & 13 & 14 & 18 & 30 & 21.1 & 28.8 & 27.5 & Left caudal ${ }^{\#}$ \\
\hline 32 & 5 & 7 & 11 & 19 & 24 & 20.4 & 25.9 & 26.2 & Left caudal, 12 \\
\hline 29 & 5 & 11 & 13 & 13 & 18 & 12 & 17.9 & 29.2 & Right caudal, 8 \\
\hline Mean士sD & & $10.5 \pm 2.5$ & $12.8 \pm 1.3$ & $16.5 \pm 2.6$ & $23.5 \pm 5$ & $17.2 \pm 4.3$ & $24.3 \pm 4.6$ & $26.0 \pm 3.5$ & \\
\hline 24 & 10 & 8 & 9 & & & 4.5 & 9.8 & 9.9 & Right middle, 13 \\
\hline 24 & 10 & 9 & 11 & 20 & 24 & 22.3 & 27.7 & 28.8 & Right middle, 5 \\
\hline Mean \pm sD & & $8 \pm 1.4$ & $10.7 \pm 1.7$ & $17.6 \pm 2.1$ & $20 \pm 3.5$ & $15.2 \pm 8$ & $18.8 \pm 8.5$ & $22.8 \pm 10.4$ & \\
\hline
\end{tabular}


a)

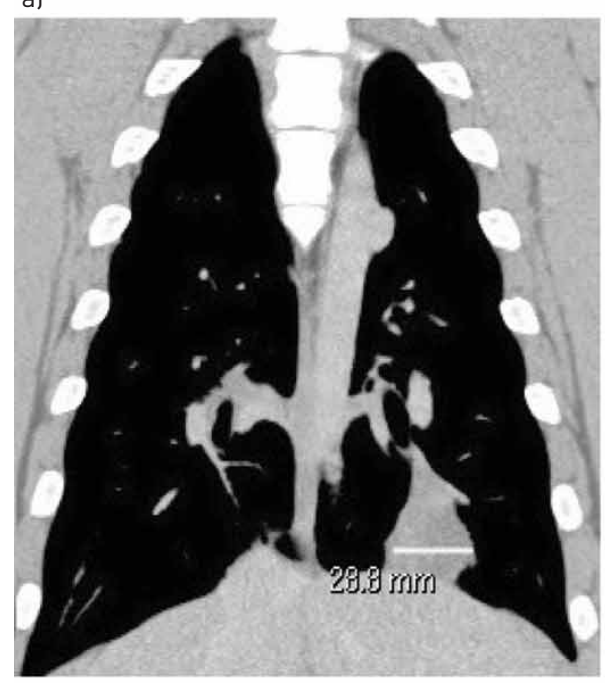

d)

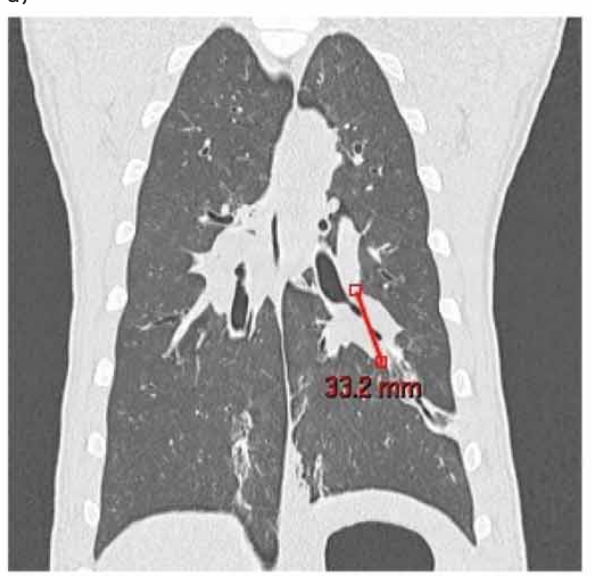

b)

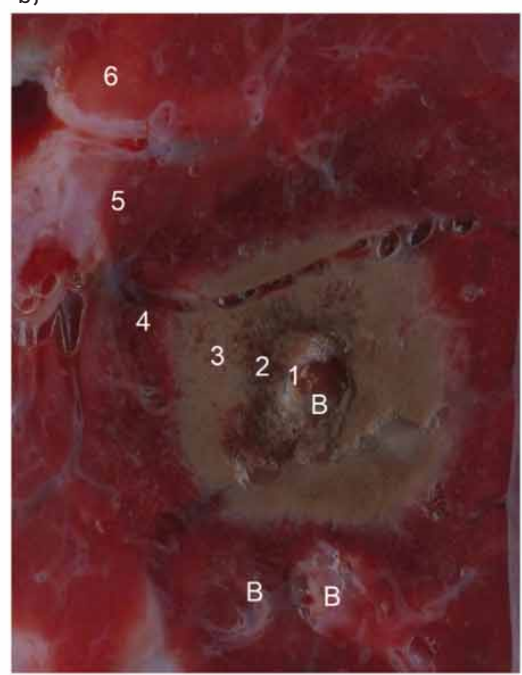

e)

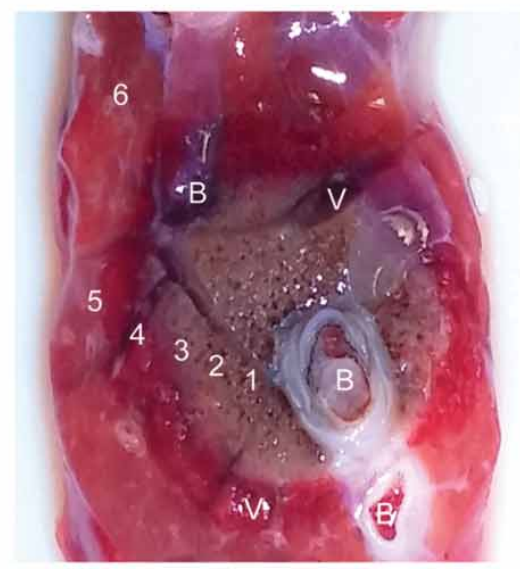

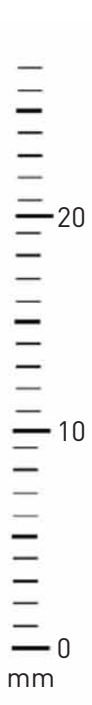

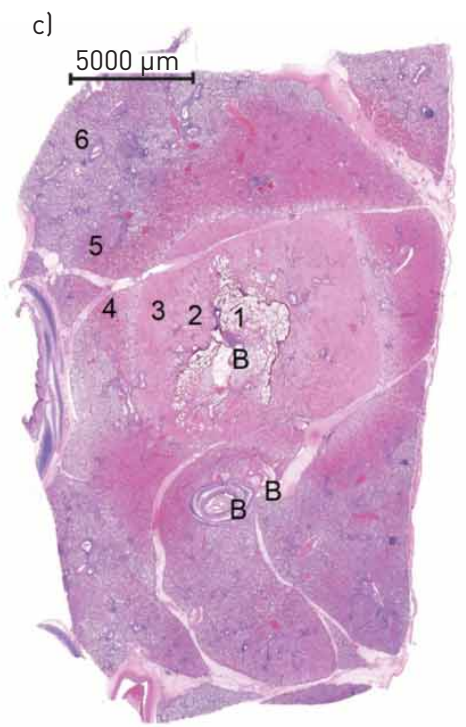

f)

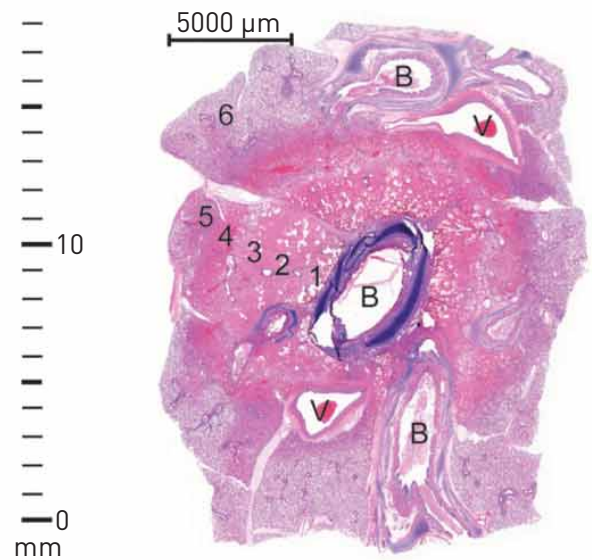

FIGURE 6 Example of computed tomography segmented ablation zones following 5 min, $32 \mathrm{~W}$ applied power (a) and for $30 \mathrm{~W}$ applied power (d). Corresponding gross images showing focal well-delineated targetoid lesion in the lung parenchyma in (b) and (c) for $32 \mathrm{~W}$ input power and (e) and ( $\mathrm{f}$ ) for $30 \mathrm{~W}$ input power. (c) (32 W) and ( $\mathrm{f})(30 \mathrm{~W})$, show corresponding haematoxylin and eosin stained sections of the lung with various zones marked 1-5 based on the histomorphology of the tissue post-ablation. "B" marks denote bronchi and "V" marks denote blood vessels. Numbers inside ablation zone represent various altered pathological changes (1-5) following ablation. Zone 6 represents unaffected lung.

present study, extents of ablation zones ranged between $16.5-23.5 \mathrm{~mm} \mathrm{(30-32} \mathrm{W,} 5 \mathrm{~min})$ and 17.6-20 $\mathrm{mm}$ $(24 \mathrm{~W}, 10 \mathrm{~min})$. Although the $32 \mathrm{~W}, 5 \mathrm{~min}$ ablations deliver lower energy $(9.6 \mathrm{~kJ})$ to tissue than the $24 \mathrm{~W}$, $10 \mathrm{~min}(14.4 \mathrm{~kJ})$ ablations, no statistically significant differences in ablation zone extent were observed. Power delivered from the ablation applicator falls offs centrifugally with increasing distance from the applicator, with the boundary of the ablation zone limited by heat sinks such as ventilation and blood perfusion. Using the higher applied power level $(32 \mathrm{~W})$ albeit for a shorter time $(5 \mathrm{~min})$ probably leads to heating by direct microwave absorption at greater distances from the applicator, compared to when using lower power $(24 \mathrm{~W})$, thereby compensating for the lower ablation duration. In previously published studies of MWA with percutaneous needle-based applicators in an in vivo porcine lung model, ablation zone extents as large as $28-38 \mathrm{~mm}$ in diameter have been reported when applying $70 \mathrm{~W}$ to a single needle-based antenna [20]. Due to considerable attenuation within the 1.1-m catheter used in the present study, only approximately half the power available at the applicator input was available at the antenna radiating tip. Thus, the power levels employed in this study were only approximately $34-45 \%$ of the power levels used in previous studies with needle-based applicators, leading to smaller ablation zones. Higher power levels were precluded as benchtop studies illustrated the potential for catheter thermal failure at power levels exceeding $32 \mathrm{~W}$ applied at the catheter tip (60 W at the catheter proximal end). Further refinements to applicator materials and coolant flow are warranted to enable use of higher power levels and thus afford creation of larger ablation zones. Another potential contributor to the smaller ablation zones observed in this study may be the heat sinks due to large blood vessels and airways in the central 


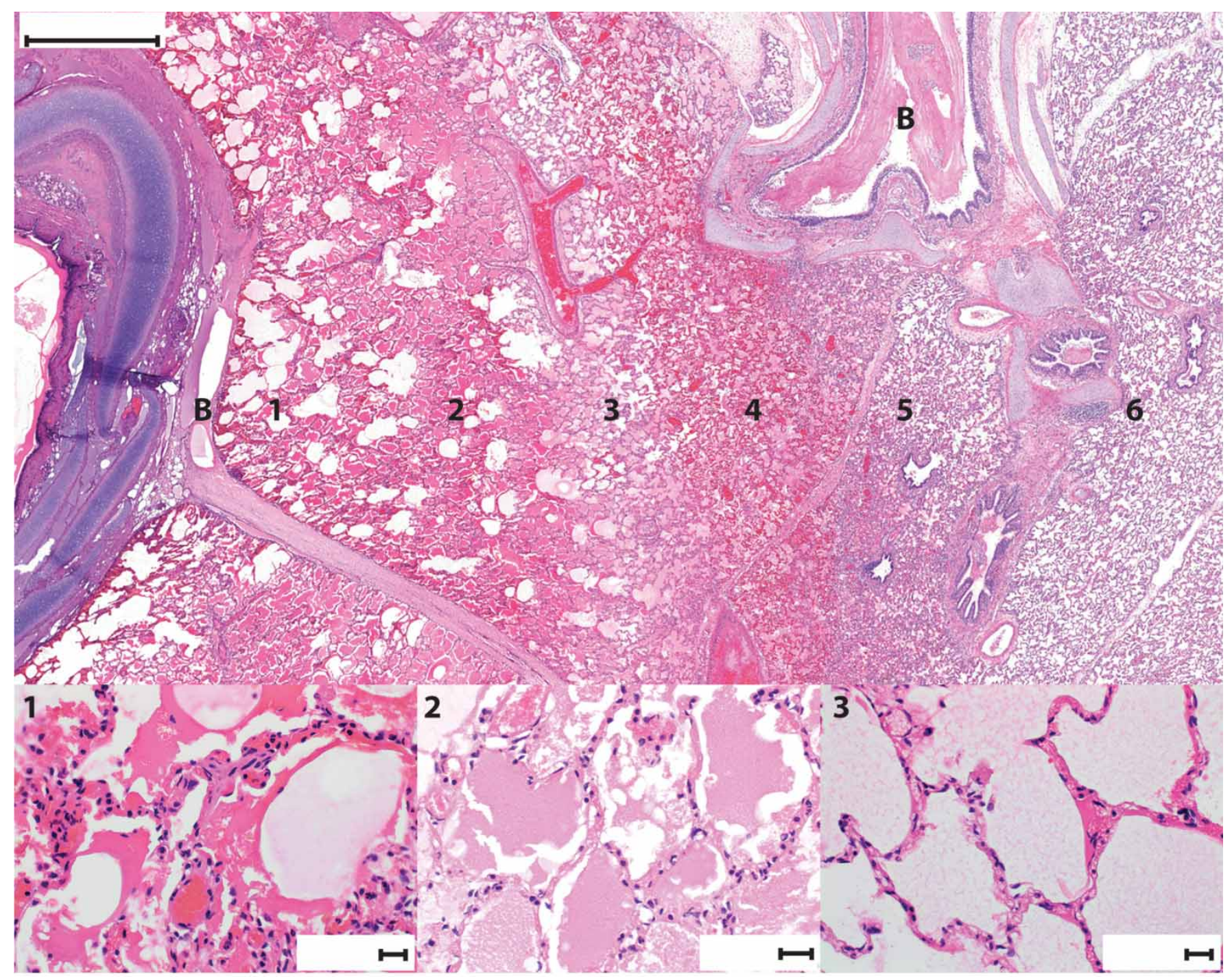

Necrosis

Oedema

Mild oedema and emphysema

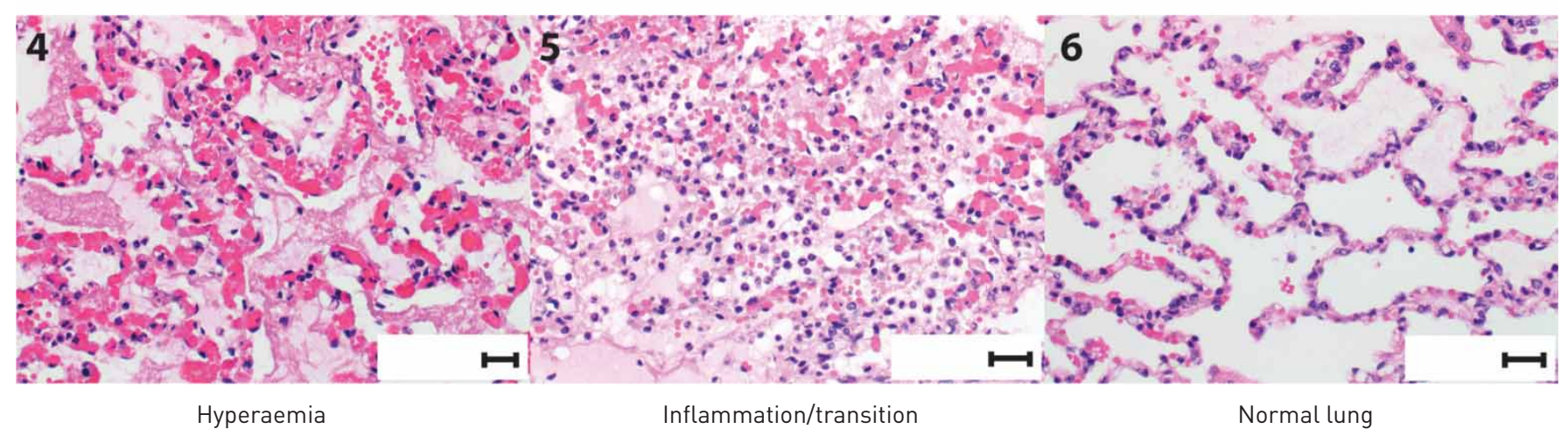

FIGURE 7 Haematoxylin and eosin stained section of the lung showing various zones (1-5) representing histopathological changes in tissue following ablation and unaffected lung (zone 6).

(hilar) region of the lung, which are recognised to contribute to smaller ablation zones compared to when the same energy is used in peripheral regions [21]. Most previous studies using needle-based microwave technologies have focused on creating ablation zones in the peripheral lung, distant from larger airways and blood vessels. Nevertheless, as illustrated in figure 6, the present study demonstrated the feasibility of creating ablation zones in the vicinity of airways. This presents a distinct advantage of microwave power, 
in contrast to radiofrequency ablation technologies, which are severely impeded by the presence of large airways.

The ablation zone sizes observed on CT post-treatment were generally in good agreement (difference of $1 \pm 3 \mathrm{~mm}$ ) with the extent of the ablation zone as assessed by the hyperaemic rim observed on gross pathology, and approximately 7-12 mm larger than the central brown zone, similar to previous reports [22]. The extent of the ablation characterised on CT provided the means for estimating volume of the ablation zone, which was not feasible with the gross pathology measurement, where determining the axis of the applicator insertion remains challenging.

Histopathological examination of the tissue excised acutely following ablation showed variability in the cellular damage from the centre of the lesion to the periphery. The intensity of tissue changes were directly proportional to the intensity of heating within the tissue, with the centre (closest to the ablation applicator) being the highest, and decreasing centrifugally. Within zone 1 ( $\sim 20 \%$ of the affected region), acute parenchymal lung necrosis was observed with loss of tissue architecture and presence of cellular and nuclear fragmentation (hypereosinophilia). The remainder of the zones (2-5) showed histopathological changes consistent with degeneration and acute inflammation. Emphysema (zone 3) is noticed when the air gets trapped in the alveolar spaces, oedema and congestion (zone 4) are often due to leaky blood vessels and increased vascular supply respectively, and the outermost inflammatory zone (zone 5) contains infiltrates of neutrophils and macrophages (to wall-off injury). It is well established that the full extent and architectural characteristics of coagulative necrosis are not apparent on histology until at least $24-48 \mathrm{~h}$ following thermal ablation [23-25]. For assessing the extent of thermal ablation during acute nonsurvival studies such as the present study, vital staining techniques that distinguish viable from nonviable tissue as a marker of mitochondrial activity have been shown to provide a more reliable assessment of the extent of the treatment zone [26]. Indeed, in human clinical studies where the ablated lung tumour and surrounding margin of tissue is surgically resected immediately following the ablation, viability stains have been employed for assessment of the treatment zone as histological assessment of the extent of necrosis is equivocal at this early timepoint [27-29]. Further studies in animals surviving $>3$ days following ablation are warranted to definitively evaluate histopathological features in zones $2-5$ at later time points, as well as to observe changes in the ablation zone as assessed on CT imaging.

The average length of tunnel paths (i.e. distance beyond the airway wall) created to reach the centre of targeted lung regions was $12 \mathrm{~mm}$, with a maximum tunnel length of $24.5 \mathrm{~mm}$ and minimum of $5 \mathrm{~mm}$. No significant bleeding into the airways was observed. To our knowledge, this is the first study reporting use of virtual bronchoscopy to guide safe delivery of MWA applicators to sites $>2 \mathrm{~cm}$ beyond the airway wall; previous reports of flexible MWA devices in the lung have only been reported in preliminary abstract form $[30,31]$. Previous pre-clinical studies (in canines) and human clinical studies have demonstrated the use of virtual bronchoscopy to deliver biopsy tools to targets in the lung parenchyma with tunnel lengths up to $48 \mathrm{~mm}$ beyond the airway wall $[12,13,15]$. The relatively small airways of porcine lung limited the range of manoeuvring the 6-mm bronchoscope and restricted tunnel length for accessible targets to be $<3 \mathrm{~cm}$.

Ablation systems with flexible applicators employing a variety of energy modalities (radiofrequency [32-34], microwave [14], laser [35]) are in development for bronchoscopic treatment of lung nodules. These systems may provide a minimally invasive approach for treatment of localised lung nodules as detected on CT screening $[11,36]$. However, both radiofrequency and laser ablation systems require the controlled energy delivery due to changes in tissue electrical and optical properties, respectively, at elevated temperatures. These changes include a sharp drop in electrical conductivity and significant rise of optical absorption in tissue at temperatures exceeding $\sim 100{ }^{\circ} \mathrm{C}$ limiting further delivery of the current in case of radiofrequency and the amount of power radiated to distant tissue regions in case of laser [37]. In contrast to these modalities, microwaves are not impeded by tissue carbonisation at elevated temperatures, and may thus enable ablation of large tissue volumes without complex energy-delivery algorithms, as previously demonstrated in pre-clinical studies with needle-based applicators [20,38]. Since thermal ablation of lung nodules requires the ablation of a peri-tumoural margin of normal tissue, the ability to heat larger tissue volumes with microwave applicators provides a theoretical advantage. Further development of our catheter will enable use of higher power levels.

A limitation of this study was that ablation zones were assessed in normal lung. Lung tumours can have considerably different electrical, thermal, blood flow and ventilation profiles compared to normal tissue. Specifically, due to greater ventilation (i.e. heat sink) within normal lung, the ablation zones reported here may serve as a lower bound for the anticipated size of ablation zones in tumours. As there are no established large animal models of lung tumours, ablation volumes observed in normal porcine lung may be used as a guide to anticipate extent of ablation zones in tumours, similar to percutaneous ablation treatment planning in current clinical use. Future studies may use injected muscle paste/gel to simulate the 
effect of solid/dense tumours within normal lung [39]. Other limitations included the relatively small number of applied power levels and ablation durations, as well as the lack of a survival study.

\section{Conclusion}

We have integrated an MWA platform, incorporating a flexible, water-cooled applicator, with a clinically established bronchoscopic navigation and guidance platform and demonstrated the technical feasibility of the system for safely ablating lung tissue in an in vivo porcine model. Ablations were performed by delivering the applicator to targets in lung parenchyma via 5-24-mm tunnels under the guidance of a bronchoscopic navigation system. No peri-procedural complications were observed. Further system development to enable use of higher power levels and pre-clinical survival studies are warranted to assess the safety and efficacy of this technology in a clinical setting.

Acknowledgements: We gratefully acknowledge Mal Hoover, KSU College of Veterinary Medicine (Manhattan, KS, USA), for help with preparing graphical illustrations; Abbe Smith and John Placho, Broncus Medical, Inc. (San Jose, CA, USA), for providing training and technical assistance with the Archimedes system; and Nick Hemphill, Veterinary Health Center, Kansas State University (Manhattan, KS, USA) for performing CT scans.

Conflict of interest: J. Sebek reports grant from the Czech Ministry of Education, Youth and Sports, during the conduct of the study. S. Kramer has nothing to disclose. R. Rocha has nothing to disclose. K-C. Yu has nothing to disclose. R. Bortel reports grants from the Czech Ministry of Education, Youth and Sports, during the conduct of the study. W.L. Beard has nothing to disclose. D.S. Biller has nothing to disclose. D.S. Hodgson has nothing to disclose. C.K. Ganta has nothing to disclose. H. Wibovo has nothing to disclose. J. Yee has nothing to disclose. R. Myers has nothing to disclose. S. Lam has nothing to disclose. P. Prakash reports grants from the National Institutes of Health during the conduct of the study; and grants from Hologic, Inc., and the National Institutes of Health, outside the submitted work. In addition, P. Prakash has a patent US 62/450,916 pending and within the past 12 months, in addition to the active grants listed above, has received grants from Neurent Medical, Ltd. and Broncus Medical, Inc., for projects unrelated to this work. These projects are now completed and no longer active.

Support statement: This work was supported by US National Institutes of Health grant R01 CA218357 and by Czech Ministry of Education, Youth and Sports OP VVV funded project "CZ.02.1.01/0.0/0.0/16_019/0000765 Research Center for Informatics". Funding information for this article has been deposited with the Crossref Funder Registry.

\section{References}

1 Gorenstein LA, Sonett JR. The surgical management of stage I and stage II lung cancer. Surg Oncol Clin 2011; 20: 701-720.

2 Mazzone P. Preoperative evaluation of the lung resection candidate. Cleve Clin J Med 2012; 79: e-Suppl. 1, e-S17-e-S22.

3 Powell JW, Dexter E, Scalzetti EM, et al. Treatment advances for medically inoperable non-small-cell lung cancer: emphasis on prospective trials. Lancet Oncol 2009; 10: 885-894.

4 Tandberg DJ, Tong BC, Ackerson BG, et al. Surgery versus stereotactic body radiation therapy for stage I non-small cell lung cancer: a comprehensive review. Cancer 2018; 124: 667-678.

5 Dupuy DE, Fernando HC, Hillman S, et al. Radiofrequency ablation of stage IA non-small cell lung cancer in medically inoperable patients: results from the American College of Surgeons Oncology Group Z4033 (Alliance) trial. Cancer 2015; 121: 3491-3498.

6 Sidoff L, Dupuy DE. Clinical experiences with microwave thermal ablation of lung malignancies. Int $J$ Hyperthermia 2017; 33: 25-33.

7 de Baere T, Tselikas L, Catena V, et al. Percutaneous thermal ablation of primary lung cancer. Diagn Interv Imaging 2016; 97: 1019-1024.

8 Brace CL. Radiofrequency and microwave ablation of the liver, lung, kidney, and bone: what are the differences? Curr Probl Diagn Radiol 2009; 38: 135-143.

9 Tsakok MT, Little MW, Hynes G, et al. Local control, safety, and survival following image-guided percutaneous microwave thermal ablation in primary lung malignancy. Clin Radiol 2019; 74: 80.e19-80.e26.

10 Izaaryene J, Cohen F, Souteyrand $\mathrm{P}$, et al. Pathological effects of lung radiofrequency ablation that contribute to pneumothorax, using a porcine model. Int J Hyperth 2017; 33: 713-716.

11 Harris K, Puchalski J, Sterman D. Recent advances in bronchoscopic treatment of peripheral lung cancers. Chest 2017; 151: 674-685.

12 Sterman DH, Keast T, Rai L, et al. High yield of bronchoscopic transparenchymal nodule access real-time image-guided sampling in a novel model of small pulmonary nodules in canines. Chest 2015; 147: 700-707.

13 Harzheim D, Sterman D, Shah PL, et al. Bronchoscopic transparenchymal nodule access: feasibility and safety in an endoscopic unit. Respir Int Rev Thorac Dis 2016; 91: 302-306.

14 Pfannenstiel A, Keast T, Kramer S, et al. Flexible microwave ablation applicator for the treatment of pulmonary malignancies. Ryan TP, ed. In: Energy-based Treatment of Tissue and Assessment IX. Proc SPIE 2017; 10066: $100660 \mathrm{M}$.

15 Silvestri GA, Herth FJ, Keast T, et al. Feasibility and safety of bronchoscopic transparenchymal nodule access in canines: a new real-time image-guided approach to lung lesions. Chest 2014; 145: 833-838.

16 Rai L, Keast TM, Wibowo H, et al. Fluoroscopic image-guided intervention system for transbronchial localization. In: Medical Imaging 2012: Image-Guided Procedures, Robotic Interventions, and Modeling. 8316: 831624. 2012, Bellingham, International Society for Optics and Photonics.

17 Durick NA, Laeseke PF, Broderick LS, et al. Microwave ablation with triaxial antennas tuned for lung: results in an in vivo porcine model. Radiology 2008; 247: 80-87. 
18 Ahmed M, Solbiati L, Brace CL, et al. Image-guided tumor ablation: standardization of terminology and reporting criteria-a 10-year update. Radiology 2014; 273: 241-260.

19 Kodama H, Ueshima E, Gao S, et al. High-power microwave ablation of normal swine lung: impact of duration of energy delivery on adverse event and heat sink effects. Int J Hyperth 2018; 34: 1186-1193.

20 Brace CL, Hinshaw JL, Laeseke PF, et al. Pulmonary thermal ablation: comparison of radiofrequency and microwave devices by using gross pathologic and CT findings in a swine model. Radiology 2009; 251: 705-711.

21 Al-Hakim RA, Abtin FG, Genshaft SJ, et al. Defining new metrics in microwave ablation of pulmonary tumors: ablation work and ablation resistance score. J Vasc Interv Radiol JVIR 2016; 27: 1380-1386.

22 Meram E, Longhurst C, Brace CL, et al. Comparison of conventional and cone-beam CT for monitoring and assessing pulmonary microwave ablation in a porcine model. J Vasc Interv Radiol JVIR 2018; 29: 1447-1454.

23 Coad JE, Bischof JC. Histologic differences between cryothermic and hyperthermic therapies. Presented at the Society of Photo-Optical Instrumentation Engineers (SPIE) Conference Series 2003; 4954: 27-36.

24 Goldberg SN, Gazelle GS, Compton CC, et al. Radio-frequency tissue ablation of VX2 tumor nodules in the rabbit lung. Acad Radiol 1996; 3: 929-935.

25 Rendon RA, Gertner MR, Sherar MD, et al. Development of a radiofrequency based thermal therapy technique in an in vivo porcine model for the treatment of small renal masses. J Urol 2001; 166: 292-298.

26 Marcovich R, Aldana JPA, Morgenstern N, et al. Optimal lesion assessment following acute radio frequency ablation of porcine kidney: cellular viability or histopathology? J Urol 2003; 170: 1370-1374.

27 Nguyen CL, Scott WJ, Young NA, et al. Radiofrequency ablation of primary lung cancer: results from an ablate and resect pilot study. Chest 2005; 128: 3507-3511.

28 Schneider T, Reuss D, Warth A, et al. The efficacy of bipolar and multipolar radiofrequency ablation of lung neoplasms - results of an ablate and resect study. Eur J Cardio-Thorac Surg 2011; 39: 968-973.

29 Schneider T, Warth A, Herpel E, et al. Intraoperative radiofrequency ablation of lung metastases and histologic evaluation. Ann Thorac Surg 2009; 87: 379-384.

30 Ferguson J, Egressy K, Schefelker R, et al. Bronchoscopically-guided microwave ablation in the lung. Chest 2013; 144: 87A.

31 Howk K, Dickhans W, Rooks K, et al. Characterization of a Bronchoscopic Thermal Ablation Catheter in Porcine Lung. Am J Respir Crit Care Med 2016; 193: A6019.

32 Safi S, op den Winkel J, Kramer S, et al. A new bronchoscopic catheter for the transbronchial ablation of pulmonary nodules. Lung Cancer Amst Neth 2018; 124: 125-129.

33 Eberhardt R, Kahn N, Herth FJF. 'Heat and destroy': bronchoscopic-guided therapy of peripheral lung lesions. Respir Int Rev Thorac Dis 2010; 79: 265-273.

34 Koizumi T, Tsushima K, Tanabe $\mathrm{T}$, et al. Bronchoscopy-guided cooled radiofrequency ablation as a novel intervention therapy for peripheral lung cancer. Respir Int Rev Thorac Dis 2015; 90: 47-55.

35 Casal RF, Walsh G, McArthur M, et al. Bronchoscopic laser interstitial thermal therapy: an experimental study in normal porcine lung parenchyma. J Bronchol Interv Pulmonol 2018; 25: 322-329.

36 Sabath BF, Casal RF. Bronchoscopic ablation of peripheral lung tumors. J Thorac Dis 2019; 11: 2628-2638.

37 Fahrenholtz SJ, Stafford RJ, Maier F, et al. Generalised polynomial chaos-based uncertainty quantification for planning MRgLITT procedures. Int J Hyperth 2013; 29: 324-335.

38 Andreano A, Huang Y, Meloni MF, et al. Microwaves create larger ablations than radiofrequency when controlled for power in ex vivo tissue. Med Phys 2010; 37: 2967-2973.

39 Planché $\mathrm{O}$, Teriitehau C, Boudabous S, et al. In vivo evaluation of lung microwave ablation in a porcine tumor mimic model. Cardiovasc Intervent Radiol 2013; 36: 221-228. 